\title{
Body perception disturbance: A contribution to pain in complex regional pain syndrome (CRPS)
}

\author{
Jennifer S. Lewis ${ }^{\mathrm{a}, \mathrm{b}, *}$, Paula Kersten ${ }^{\mathrm{b}}$, Candida S. McCabe ${ }^{\mathrm{a}, \mathrm{c}}$, \\ Kathryn M. McPherson ${ }^{\mathrm{d}}$, David R. Blake ${ }^{\mathrm{a}, \mathrm{c}}$ \\ a The Royal National Hospital for Rheumatic Diseases NHS Foundation Trust, Bath, UK \\ b The School for Health Professions and Rehabilitation Sciences, University of Southampton, Southampton, UK \\ c School for Health, University of Bath, Bath, UK \\ d Auckland University of Technology, Auckland, New Zealand
}

Received 16 August 2006; received in revised form 6 February 2007; accepted 12 March 2007

\section{Abstract}

In spite of pain in the CRPS limb, clinical observations show patients pay little attention to, and fail to care for, their affected limb as if it were not part of their body. Literature describes this phenomenon in terms of neurological neglect-like symptoms. This qualitative study sought to explore the nature of this phenomenon with a view to providing insights into central mechanisms and the relationship with pain. Twenty-seven participants who met the IASP CRPS classification were interviewed using qualitative methods to explore feelings and perceptions about their affected body parts. These semi-structured interviews were analysed utilising principles of grounded theory. Participants revealed bizarre perceptions about a part of their body and expressed a desperate desire to amputate this part despite the prospect of further pain and functional loss. A mismatch was experienced between the sensation of the limb and how it looked. Anatomical parts of the CRPS limb were erased in mental representations of the affected area. Pain generated a raised consciousness of the limb yet there was a lack of awareness as to its position. These feelings were about the CRPS limb only as the remaining unaffected body was felt to be normal. Findings suggest that there is a complex interaction between pain, disturbances in body perception and central remapping. Clinically, findings support the use of treatments that target cortical areas, which may reduce body perception disturbance and pain. We propose that body perception disturbance is a more appropriate term than 'neglect-like' symptoms to describe this phenomenon.

(C) 2007 Published by Elsevier B.V. on behalf of International Association for the Study of Pain.

Keywords: Complex regional pain syndrome; Reflex sympathetic dystrophy; Patient experience; Body perception disturbance; Mental body representation; Pain; Neglect; Central mechanisms

\section{Introduction}

Pain in a limb is the cardinal symptom of complex regional pain syndrome (CRPS). One could assume therefore that patients might be overattentive, wishing to protect or look after their affected limb. However,

\footnotetext{
"Corresponding author. Address: The Royal National Hospital for Rheumatic Diseases NHS Foundation Trust, Upper Borough Walls, Bath BA1 1Rl, UK. Tel.: +441225787043; fax: +441225463223.

E-mail address: Jenny.lewis@rnhrd-tr.swest.nhs.uk (J.S. Lewis).
}

both clinical observations and recent literature (Galer 35 et al., 1995; Galer and Jensen, 1999; Lewis et al., 2003) 36 suggest that this is not the case. Patients have been 37 reported to neglect their affected limb despite the pain. 38 They do not attend to their limb often positioning it in 39 such a way that it is outside of their field of view (Lewis 40 et al., 2003). Galer et al. $(1995,1999)$ proposed that this 41 phenomenon was similar in form to neurological 42 neglect. Some consider their affected hand as foreign 43 or strange (Forderreuther et al., 2004) and larger than 44 it really is (Moseley, 2005). It is clear from these 45 
46 reported observations that patient's behaviour towards

their affected limb is altered but quite how an individual with CRPS perceives their affected body parts is not well understood.

Recent brain imaging studies (Maihofner et al., 2003; Pleger et al., 2005) have shown that those with CRPS have a disrupted cortical map of the affected body part. An association between symptom resolution over time and the corrective reorganisation of cortical limb representation suggests that there is a relationship between the amount of pain and the degree of cortical disorganisation (Flor, 2003; Maihofner et al., 2004; Pleger et al., 2005). The existence of these disturbed representations could serve to influence the pain experience and alter how an individual may perceive their CRPS limb. In order to better understand these representations and their impact, it is important to know how people with CRPS actually perceive their own affected body parts. A review of the literature suggests that to date, this perspective remains poorly researched and poorly understood. We suggest a greater knowledge of body perception in CRPS may provide valuable insights into central mechanisms and the relationship with pain. In addition, given that CRPS is known to be resistive to conventional treatments, an improved understanding of body perception in relation to pain may identify areas in which interventions could be specifically targeted.

To achieve these objectives, we set out to explore the patients' experience of body perception in CRPS, to postulate a theoretical framework for understanding the characteristics of body perception disturbance and how it may contribute to the pain experience. Finally, we discuss how these findings may inform clinical practice.

\section{Methods}

Given the inductive nature of this research (i.e. hypothesis deriving not hypothesis testing), a qualitative methodology, drawing on principles of grounded theory, was utilised. This approach, previously used in the pain field, is of particular value in exploring areas such as this, where little research has been undertaken and knowledge is limited (Flick, 2002). Just as case studies are used to inform and develop hypotheses, a strength of grounded theory is that it aims to generate theory from the data from which new hypotheses can be made. Emergent theory is not merely descriptive but seeks to identify key relationships within and between both existing and newly proposed theory in order to enhance our understanding (Glaser, 1969; Strauss and Corbin, 1998; Seale, 1999; Stanley and Cheek, 2003).

\subsection{Participants and data collection}

Adult participants who met The International Association of the Study of Pain classification criteria for CRPS Type I and II (Stanton-Hicks et al., 1995) were drawn from a UK population.
Additional inclusion criteria were the capability to verbally communicate and the absence of co-morbidity such as diabetic neuropathy which may significantly influence their experience. Recruitment sources included a national CRPS referral hospital, RSD-UK, the United Kingdom CRPS patient charity, pain clinics and an orthopaedic department. Purposive sampling was initially employed to gain a broad spectrum of experiences using the following variables: age, gender, disease duration and body part affected. Subsequently, participants were selected in order to refine and clarify emergent theories (Theoretical sampling: Glaser, 1978). Sampling continued until the data reached saturation whereby no new themes emerged.

Multi Centre Research Ethics Committee and relevant NHS Trust approvals were granted prior to commencing data collection.

Following written informed consent, qualitative data regarding participants' experience of CRPS were collected using semi-structured interviews. An interview schedule of open-ended questions was used to explore perceptions about the affected limb, how it looked and how it felt both physically and emotionally. The aim was to elicit detailed descriptions with a view to gaining in-depth experience from the participant's perspective and hence a better understanding of this phenomenon (Denzin and Lincoln, 1998). Interviews were undertaken in the participant's own home to encourage a feeling of relaxation and openness. Themes that emerged through the analysis of the early interviews informed the schedule for subsequent interviews. Memos were taken during and after the interviews documenting ideas and emerging theories as the interviews progressed.

\subsection{Measures}

In order to describe the study sample within the context of the general CRPS population it was appropriate to measure pain, depression and health status. Both the McGill Pain questionnaire and Brief Pain Inventory were considered too long and burdensome, therefore the short form Brief Pain Inventory (BPI: Cleeland and Ryan, 1994; Cleeland et al., 1996) was chosen. The inventory measures pain intensity and interference by participants rating (on a scale of $0-10$ ) three factors: (a) their current pain, (b) pain intensity over the previous week and (c) the degree to which pain has interfered with physical, social and psychological aspects of functioning.

Many people with pain suffer from depression. Commonly used in pain studies, the Beck Depression Inventory (BDI: Beck et al., 1961), although slightly longer than the HADS (Zigmond and Snaith, 1983), was considered suitable. Given the exploratory nature of the study, the depth of the BDI was seen as a strength over a screening tool such as the HADS (Love et al., 2004). The BDI evaluates 21 depressive symptoms on a four point intensity scale comprising emotional, behavioural and somatic symptoms. The SF-36 (Ware and Sherbourne, 1992) was chosen to measure health status. Although this measure is generic and some aspects are not specific to this population it was considered important to reflect the health status of the study sample and gave the potential for comparing with normative data.

Questionnaire and assessment tools were administered following the interview so as not to influence interview responses. 


\subsection{Data analysis}

158 Interviews were recorded and transcribed verbatim. To pro159 tect anonymity, participant names were replaced with identifi160 cation codes. Interviewees checked the transcripts to ensure 161 reliability of the data. Manual data analysis was initially 162 undertaken followed by further in depth analysis with the 163 aid of a data software program (QSR NVivo (Richards, 164 2002)). Significant statements from the initial interviews were 165 grouped together into topic areas. In depth questions about 166 these areas were asked at subsequent interviews to form preli167 minary theory. Cases challenging the emerging theory (nega168 tive case analysis: Glaser, 1978) were sought to develop and 169 redefine the theory. A peer analysis review involving the exam170 ination of data by qualitative experts was undertaken to ensure 171 that data analysis was credible and robust. Such steps may be 172 considered similar to the requirement in quantitative research 173 to demonstrate the reliability and validity of data.

174 Data from the questionnaires were statistically analysed 175 using SPSS version 12.0.1 for windows (SPSS, 2003).

\section{3. Results}

\section{3.1. Pain, quality of life and depression measures}

178 Results from the BPI, BDI and SF-36 are illustrated 179 in Table 2. As the sample size is small, median scores 180 and interquartile ranges (IQR) have been used rather 181 than means and confidence intervals as is more common with these data. Whilst caution is necessary given the small sample and wide variation of scores, the sample can be seen to have moderate pain intensity and pain interference (BPI). They were physically limited but with no mental limitations (SF-36) and had mild to moderate depression ratings (BDI).

Twenty-seven participants (12 males, 15 females) were interviewed generating descriptions about body perception experience of considerable scope including those relating to pain. Six themes encapsulated the descriptions of that experience. Themes were noted if they were either repeated by a number of participants or because of the strength of feeling with which they were spoken. These are described and supported with quotes from the interviews. Participant identification codes are given in brackets at the end of each quote as noted below in Table 1. Pain is described in context within the themes where relevant.

The themes are as follows: hostile feelings, spectrum of disassociation; disparity between what is apparent and what is felt; distorted mental image of affected part; awareness of limb position; conscious attention.

The two initial themes are concerned with the attitudes and emotions that were expressed about the affected body part. More abstract perceptions about the appearance and inner felt sense of the affected limb are described in the remaining themes.

Table 1

Participant demographics

\begin{tabular}{|c|c|c|c|c|c|c|}
\hline Participant ID code & Gender & Age at interview & CRPS type & CRPS trigger & Body part affected & Duration of condition (years) \\
\hline 1 & Male & 38 & $\mathrm{I}$ & Soft tissue & Upper limb & 5 \\
\hline 2 & Male & 51 & I & Soft tissue & Upper limb & 3.5 \\
\hline 3 & Male & 50 & I & Fracture & Upper limb & 3 \\
\hline 4 & Female & 23 & I & Post injection & Lower limb & 8 \\
\hline 5 & Female & 51 & I & Spontaneous & Both & 13 \\
\hline 6 & Female & 51 & I & Spontaneous & Both & 15 \\
\hline 7 & Male & 42 & $\mathrm{I}$ & Soft tissue & Lower limb & 4 \\
\hline 8 & Male & 68 & I & Soft tissue & Upper limb & 21 \\
\hline 9 & Female & 26 & $\mathrm{I}$ & Soft tissue & Lower limb & 8 \\
\hline 10 & Male & 56 & $\mathrm{I}$ & Post surgery & Lower limb & 13 \\
\hline 11 & Female & 43 & II & Post surgery & Upper limb & 8 \\
\hline 12 & Female & 18 & $\mathrm{I}$ & Soft tissue & Both & 10 \\
\hline 13 & Male & 19 & $\mathrm{I}$ & Soft tissue & Lower limb & 8 \\
\hline 14 & Female & 47 & $\mathrm{I}$ & Fracture & Upper limb & 0.25 \\
\hline 15 & Male & 38 & II & Soft tissue & Upper limb & 1.5 \\
\hline 16 & Male & 33 & $\mathrm{I}$ & Post surgery & Upper limb & 2 \\
\hline 17 & Female & 34 & $\mathrm{I}$ & Spontaneous & Upper limb & 5 \\
\hline 18 & Female & 61 & I & Fracture & Upper limb & 0.25 \\
\hline 19 & Female & 51 & I & Fracture & Lower limb & 8 \\
\hline 20 & Female & 53 & I & Post surgery & Lower limb & 4 \\
\hline 21 & Female & 40 & I & Soft tissue & Lower limb & 0.4 \\
\hline 22 & Male & 45 & I & Fracture & Upper limb & 1.25 \\
\hline 23 & Female & 26 & I & Soft tissue & Lower limb & 8 \\
\hline 24 & Female & 49 & $\mathrm{I}$ & Spontaneous & Upper limb & 4 \\
\hline 25 & Male & 37 & I & Spontaneous & Lower limb & 7 \\
\hline 26 & Female & 43 & $\mathrm{I}$ & Soft tissue & Lower limb & 1.2 \\
\hline 27 & Male & 62 & $\mathrm{I}$ & Post shingles & Lower limb & 1 \\
\hline
\end{tabular}


Table 2

Pain, health status and depression measures

\begin{tabular}{llll}
\hline Measure & Median (IQR) & Mean (SD) & Range \\
\hline Brief Pain Inventory (BPI) (Short form) & & & $6(1.5)$ \\
$\quad$ Pain intensity & $6.25(5.25-7.1)$ & $6.21(2.82)$ & $3-8.5$ \\
$\quad$ Pain interference & $7.14(4.7-8.35)$ & & $0.14-10$ \\
SF-36 & & $33.1(31)$ & \\
$\quad$ Physical scale & $20(5-65)$ & $54.1(22.3)$ & $0-90$ \\
$\quad$ Mental scale & $52(36-68)$ & $21.9(14.8)$ & $16-96$ \\
Beck Depression Inventory (BDI) & $19.5(10.25-33.5)$ & $0-57$ \\
\hline
\end{tabular}

\subsubsection{Hostile feelings}

The majority of participants described hostile feelings about the affected limb whilst unaffected parts were felt to be normal. The intensity of feeling was expressed in varying degrees irrespective of disease duration. These feelings ranged from mild frustration to a considerably more intense experience of hate disgust and repulsion;

"I feel disgust, I know it sounds a very strong word to use but I'm disgusted that my arm is this way." (15)

Participants explained that one of the reasons they had negative feelings was because their pain and other symptoms prevented them doing what they were once able. They blamed the affected limb as the cause and as such centred anger and hate towards it.

"I got more cross with it, like if you pick up a bottle, I picked up a bottle the other day from the step and it dropped straight out of my hand only because I hadn't gripped it as I thought I'd gripped it." (18)

However, this was not the case for everybody as the following quote illustrates;

"I don't think, I sort of centralise my annoyance at the pain, blaming my actual leg. I don't know. I don't think I have any feelings towards it." (4)

It was clear from the descriptions that these hostile feelings changed over time. Some expressed that their negative feelings eventually diminished, whilst others reported an increase in intensity during the course of the disease;

"It's got worse, yeah I'd say it's got worse, like I've got, I think I've got more self-conscious about them [um] I hate them more kind of thing." (13)

\subsubsection{Spectrum of disassociation}

Participants spoke of how much the affected limb felt a part of their body. A spectrum of disassociation emerged from the data such that many participants described that psychologically the affected limb felt detached to varying degrees from the remainder of their unaffected body. The spectrum ranged from no feelings of detachment to an extreme form in the desperate desire of wanting to get rid of the limb.

"It was just like this foreign body you were carrying 253 around with you cause it didn't feel like it was part of you." (17)

Other participants described a stronger sense of disassociation such that the limb felt a separate entity from the body with its own control system that rebelled against the body's intentions.

'On a good day it's, it's fine, it's behaving itself you know. On a bad day that feeling comes back to me and I'd go through a period throughout the day where I feel like it's taking control and then I have to sort of muster myself and think, "No, I'm in control" so you know it's different from day to day and just sometimes if it creeps up on you it sort of overpowers you." (20)

At the severe end of the disassociation spectrum was the extreme desire of wanting to get rid of the pain by removing the affected limb. This was a common view spontaneously described by many participants who talked in graphic detail of a strong yearning to "chop off' the affected limb;

"I've sometimes felt if I could get an axe and chop it off I would do because to me as it stands, at this minute in time sat here it's a useless, it's a useless thing." (2)

Despite the commonality of this experience, a few participants felt differently as noted in the following excerpt;

"It all feels, still feels a part of my body, but, yeah, it does." (27)

\subsubsection{Disparity between what is apparent and what is felt}

Participants described how the affected part felt in relation to how it looked whilst viewing their affected limb. Many participants reported that what they saw was often at variance with how the limb felt. Rather 
289 than a general distortion, feelings of pain, size, and tem290 perature in discrete parts of the limb were discordant 291 with how their limb appeared. Intense pain was widely 292 described as being a spontaneous felt experience yet 293 not explained by the appearance of the affected part;

"At the moment my hand feels like it, the worse sort of burning that I can imagine and yet I can look at my hand and say there's nothing burning it." (15)

The size of the limb was commonly felt to be larger than its' actual appearance;

"It feels like it's really, really fat. I mean sometimes I actually look at it to, cause I think god my leg's swollen and then I'll look actually look at my leg and I think oh not it's not but it feels like it is." (26)

Pain was felt to be a reason for excessive pressure in the affected limb;

"It's almost as though there's too much pain to fit into one limb and it does feel like it's the pain that's trying to get out." (11)

Some explained there was no size disparity between the appearance of the limb and how it felt;

"That swelling's not there any more and the foot's gone back to the same size as the other one and I haven't got that sensation of it feeling larger than the other one." (06)

Participants discussed the spontaneous felt experience of temperature and how that was at odds with the temperature when touching the limb;

"Although my leg can sometimes feel cold to the touch, to me it's absolutely burning. I can literally feel that my legs are on fire. [um] But if you were to come along and touch them, then they would feel ice cold." (09)

They found these mismatches between what they were seeing and what they were feeling as confusing and distressing, as they were unable to make sense of them.

"It was just as though the, the sensations were totally mixed up. What I was seeing wasn't what I was feeling and that, that was, looking back it was confusing." (10)

Furthermore, participants described receiving a negative reaction from professionals when sharing these experiences.

"All the different types of sensations and feelings and everything you get... when you explain it to a specialist that doesn't know about this, I mean, they just look at 338 you and think, "Well, you know, you're pretty stupid." (1) 339

\subsubsection{Distorted mental image of affected parts}

Participants detailed a very distorted image when 341 asked to describe with their eyes closed, a mental picture 342 of their affected limb in comparison to their unaffected 343 contralateral limb. These imagined representations were 344 very different to the participant's descriptions of actual 345 appearance whilst looking at the affected limb. Typi- 346 cally, discrete parts rather than the whole limb were dis- 347 torted particularly in size;

"My leg, from my thigh to my knee, it feels almost a nor- 350 mal size, it does hurt, my knee feels swollen and then as I 351 get from the calf to my ankle, the swelling gets worse and 352 then from my ankle to my toes, then it feels enormous." 353 (19)

Similarly, there was a distortion in shape. Partici- 355 pants talked about a change in anatomical proportions 356 such that parts of the limb were seen as foreshortened; 357

"They actually feel as if my finger tips are me knuckles, that doesn't make sense. They, they feel a lot shorter than they should be." (22)

More intriguingly however, some described that specific parts of their affected limb were missing from their mental image;

"It's bigger than this leg (gestures to left leg) and then the rest is completely void, there's nothing there at all, I can actually see the floor. I can see a big toe and I can't see anything else from my knee down." (26)

365

366 367 368 369

By contrast, a few participants had a normal mental 370 image of their affected limb;

"Both my arms are the same colour there's no difference 373 there [um] my hands are probably both the same as well." (17)

\subsubsection{Awareness of limb position}

Participants explained that their awareness of the 378 affected limb was heightened, particularly as a conse- 379 quence of the pain and they were conscious of its pres- 380 ence. Paradoxically though, participants typically 381 expressed a difficulty in knowing its position;

"Even if, it gets to the point where if the pain is very intense, 384 even if I am touching something else I've got no idea where 385 my arm exists. The first time I realised it I think is when I 386 
woke up in bed and it, I, I just couldn't, I didn't know where my right arm was, I could feel my left arm fine but, and it wasn't like the numbness like you've rolled over and it's gone to sleep, I just had no concept of where my arm was and it was like a feeling of panic." (15)

Furthermore, when their attention was drawn to the position of the limb they became aware of how abnormal the position appeared to be as expressed by participant 11 with CRPS of the left arm.

"It's strange actually because I didn't realise until a few weeks ago what I'd been doing and I had actually been putting it behind my back a lot. Keeping it out of the way, partly because I didn't have to look at it. I wasn't aware of that until a few weeks ago when somebody mentioned it." (11)

Participants found the conflicting experience of a heightened awareness of the limb and disorientation to its position to be inexplicable and therefore confusing.

"Often I feel I'm going mad, totally and utterly bonkers because a lot of you, the logical part of you is saying this isn't and yet part of you believes it is. It's almost like you're split in two, part of you is trying to deal with it on a very logical level and the other part is dealing with how you actually feel about it." (9)

By contrast, a few participants did not describe a mismatch as they were clear that the felt position and the actual limb position were consistent;

\section{"They feel pretty much as to where they are." (21)}

\subsubsection{Conscious attention}

The last category describes the level of conscious attention participants paid to their affected body parts in terms of thinking about, looking and touching them. Thinking about the limb, for some, generated such strong feelings of disgust that they avoided doing so;

"You're watching television you see, say, a paedophile and there's something in you which is almost gut wrenching, you can't understand how an adult would treat a child like that or a young baby like that or abuse cases. That is about as close as you can get to disgust, and that's how I feel about my arm. If I can avoid thinking about it, I will." (9)

Some explained that they consciously distracted their attention away from the limb as a way of dealing with the pain;

"The pain side of it was there so I used to probably distract myself with other things and try and forget about it as much I could which in the end wasn't a very helpful idea because it, it did you do actually, I did forget about it as much as it just wasn't part of me eventually." (23)

Moreover, participants explained that they had to intensely concentrate on their limb in order to undertake what they thought to be a straightforward movement;

"If I went to move a finger, two or three fingers would move. And, it wasn't like, you know, move that finger and that happened, it was move that finger and, and this went down and you thought, "Well it should be that one." So I felt like my hand wasn't attached to my brain, if you know what I mean. It felt like I really had to concentrate to get that arm going, to get that hand going, to get that arm going. And I did have to concentrate. And it was, it was a very hard thing to do." (3)

Many participants spoke of a difficulty in paying visual attention to their limb. They avoided looking at it and in some cases deliberately hid their limb from their field of view;

"I used to try and hide it. [Um] If I was sat over there, which is where I normally sit, I would have my arm on the other side of that cushion so I couldn't see it. I still do that now. So, if I can't see it I can't be angry with it." (3)

Difficulty in touching the limb was also expressed and was mainly due to the possibility of causing pain;

"It really was very, very hard to do it. when I touched it, in lots of ways it actually reinforced the idea that it wasn't mine at all because it didn't feel like it and I couldn't make it feel like mine and I didn't want to make it feel like mine in a lot of ways because it hurt so much." (23)

By contrast some touched their limb routinely to check the limb in order to gauge a sense of normality;

"I touch it everyday to check that it still feels the same as the other foot." (21)

Participants spoke about how they were encouraged to think about, look at and touch their limb as part of rehabilitation and how this helped the process of reengaging with the limb and perceiving it in a more normal way. For example, desensitisation, a rehabilitation technique of touching the limb through stroking, massage and with the use of different textures was experienced as being beneficial;

"I started to touch it, just gentle touching for a second and I, I've built it up and built it up so that I then could massage it and talk to it and love it and accept that it was still 
488 part of me even though it was still painful, I was still angry 489 with it but I felt like I took control." (20)

490 In summary, our participants expressed strong nega491 tive feelings about a part of their own body and there 492 was a desperate desire to amputate this part despite 493 the prospect of further pain and loss of function. A mis494 match was experienced between the sensation of the $495 \mathrm{limb}$ and how it looked. Anatomical parts of the CRPS 496 limb were erased in mental representations of the 497 affected area. Pain generated a raised consciousness of 498 the limb yet there was a lack of awareness as to its posi499 tion. Participants voiced a deep tension as emotion 500 fought with logic in trying to make sense of what was 501 happening.

502 All participants described some form of disturbance 503 in how they perceived their affected body parts regard504 less of age, gender, trigger or disease duration. That is 505 to say that the presence of disturbance did not seem to 506 be associated with any one of these factors.

507 However, those with mild disease described fewer 508 components of the themes suggesting that the extent of 509 disturbance was less than those with more severe dis510 ease. Those with resolving disease spoke about feelings 511 of disassociation being more apparent during periods 512 when the disease was more active. When talking about 513 the acute stages of onset, participants mentioned being 514 aware of disturbances in body perception within as 515 few as four days of symptom presentation.

\section{4. Discussion}

517 This study has extended our understanding of dis518 turbed body perception and its contribution to pain in 519 CRPS by detailing intriguing insights from the patients' 520 perspective adding to previous studies in this area (Galer 521 et al., 1995; Galer and Jensen, 1999; Forderreuther 522 et al., 2004). Although the methodology and size of 523 the sample preclude generalisation to the total CRPS 524 population (as a quantitative study seeks to achieve), 525 findings reveal how people with this condition perceive 526 their affected limbs. Our data illustrate the bizarre atti527 tudes patients have about parts of their own body, 528 regardless of gender, disease duration and affected body 529 part, and for most, in the absence of major nerve dam530 age. Further to feeling foreign or strange, as Forderreu531 ther et al. and Galer relate $(1995,1999,2004)$ the CRPS 532 limb was felt to be another entity and the whole of the 533 affected limb was difficult for sufferers to accurately 534 locate, rather than solely a mislocation of the fingers 535 as previously described (Forderreuther et al., 2004).

536 To put these strange phenomena in the context of 537 other conditions where body perception disturbances 538 are reported, Galer has previously suggested that they 539 are similar to those seen in neurological neglect. He pro540 posed the term 'motor neglect' (Galer et al., 1995; Galer and Jensen, 1999) to describe difficulty in initiating and coordinating, and the need for mental and visual attention when moving the CRPS limb. A parallel can be drawn with our category 'conscious attention' as participants described the need for intense concentration when undertaking movements which were often difficult and inaccurate. Furthermore, cognitive neglect (Galer et al., 1995; Galer and Jensen, 1999) was reflected in aspects of our category 'spectrum of disassociation'. Participants described the limb as feeling as if it were not part of their body similar to Galer's notion of the limb not being part of their being. However, despite these similarities our findings do not wholly support Galer's hypothesis that body perception disturbances seen in CRPS mimic the traditional definition of neurological neglect-like syndrome (Galer et al., 1995; Galer and Jensen, 1999). Our data suggest that there are three important differences. First, body perception disturbances were experienced in both the acute and chronic stages whereas traditional neurological neglect is observable in the acute stages of a central lesion and often resolves spontaneously within a few weeks (Cutting, 1978). Second, participants had some insight into these disturbances, which is uncommon in neurological neglect, and finally, two participants had both upper and lower limb involvement in contralateral quadrants, whereas neurological neglect is commonly unilateral.

Our participants' descriptions could be suggestive of similarities to those seen in body dysmorphic disorder (Phillips, 1991), where patients have an imagined or minor defect in their physical appearance leading to a dislike of that area. Severe cases can result in corrective surgery to an otherwise normal body part. Our participants also described a perceived distortion of their affected limb, exacerbated when imagining it with their eyes closed, alongside a strong dislike and a desire for surgical removal of that limb. The important difference between the two conditions is that in body dysmorphic disorder the perceived distorted body part has never appeared abnormal to the external observer, whereas in CRPS changes in colour, size and temperature may have all been observed.

Unlike eating disorders where patients have a distortion in the experience of overall body shape and weight (Skrzypek et al., 2001) our participants described a distortion in the CRPS affected body part only, whilst unaffected areas were perceived as normal. Furthermore, these distortions were experienced as a variety of sensations and were more specific to discrete areas of the CRPS limb than previous studies would suggest (Moseley, 2005).

Given the discrepancies in the perception anomalies reported by our study population and those from other conditions, we suggest that rather than 'neglect-like' symptoms, the term body perception disturbance may better describe the phenomenon in CRPS. 
597 Besides the generation of novel theory, this qualita598 tive approach enables the exploration of new links to 599 existing theory. For example, one might propose that 600 the desire for amputation of an otherwise healthy limb, 601 as reported by the majority of our population, is similar 602 to autotomy (autos meaning self and totos meaning cut). 603 Although the nosological appropriateness of this term is 604 under debate (Devor, in press; Wilkie et al., in press), it 605 was defined by Patrick Wall et al. (1979) to describe the 606 maiming behaviour of rodents when they gnawed their 607 otherwise healthy, anaesthetised limb following nerve 608 ligature. Descriptions of a desire for intentional self 609 mutilation to an existing painful part appear to contra610 vene the need to guard and protect the limb against gen611 erating further pain and perhaps illustrate a modified 612 version of animal autotomy. As only two participants 613 had demonstrable nerve damage this theory has limita614 tions. However, there does appear to be a hereditary sin615 gle gene autosomal recessive trait within animal 616 autotomy (Devor and Raber, 1990). If this is the case, 617 it may indicate that a centrally driven reflex underlies 618 the onset of body perception disturbance in CRPS. Fur619 ther research is required to define whether this is genet620 ically derived.

621 A reduction in body perception disturbance appeared 622 to parallel symptom reduction, in particular pain, as 623 those with resolving disease expressed these disturbances 624 to a lesser extent. Brain imaging studies (Maihofner 625 et al., 2004; Pleger et al., 2005) demonstrating that cor626 rective cortical reorganisation correlates with a reduc627 tion in CRPS pain provide reasonable evidence to 628 suggest that there is a close relationship between altered 629 central limb representation and a disturbance in affected 630 limb perception. The relationship between pain and 631 body perception disturbance is however, more elusive. 632 Given that disturbances in body perception become 633 apparent within a few days of symptom onset and 634 appear to fluctuate in line with the intensity of symp635 toms this would suggest that possible central reorganisa636 tion is dynamic and that a complex interaction between 637 body perception disturbance and pain exists. Whether a 638 disturbance in body perception precipitates or perpetu639 ates pain remains unclear.

640 The erasing of discrete anatomical parts of the CRPS 641 limb from the mental body representation suggests a 642 specific alteration in the central body schema map. Par643 ticipants expressed surprise when describing these men644 tal distortions indicating that these changes occurred 645 outside of their conscious state of awareness. This find646 ing further supports the view that an altered central rep647 resentation contributes to a disturbance in body 648 perception. An intact cortical body schema is an essen649 tial component of movement control (Graziano and 650 Botvinick, 2002) therefore absence of limb segments 651 within the central representation of the CRPS limb 652 may have implications for function.
Participants expressed more difficulty in articulating the subtler aspects of body perception disturbances than 6 when describing pain and spoke of an inner turmoil in 6 attempting to make sense of them. Raising such concerns with health professionals may cause distress for fear of being regarded as absurd and possibly dismissed. However, many participants expressed relief at being able to share these previously untold experiences and we would suggest that clinicians encourage and reassure patients when discussing these issues, as they are clearly not alone in experiencing disturbing thoughts and feelings. Furthermore, gaining an understanding of the individuals' body perception disturbance may inform treatment choice.

As our findings suggest a close relationship between body perception disturbance and altered central representations, it would seem reasonable to target treatments at correcting this remapping with the aim of reducing pain and normalising limb perception. Novel treatments for CRPS such as mirror visual feedback (McCabe et al., 2003) and motor imagery (Moseley, 2004, 2006) are designed to target cortical areas, and appear to provide pain relief. Yet they may also be influencing other, as yet unmeasured, symptoms associated with body perception.

Participants expressed that other less well researched interventions such as desensitisation helped them to perceive their limb in a more normal way. As these strategies involve looking, touching and thinking about the affected limb they too may have an influence on central areas.

Recommendations for further research include developing a clinical tool to measure the extent and nature of body perception disturbance in CRPS. Testing the potential for new interventions in reducing body perception disturbance, as well as pain, is warranted. Such testing may also establish the efficacy of existing rehabilitation techniques. A more definitive relationship between body perception disturbance and pain could be established by determining whether CRPS patients with no ongoing pain have a disturbance in body perception.

In conclusion, this qualitative study has provided a more detailed insight into how patients with CRPS perceive the body perception anomalies associated with their affected limbs. We have demonstrated that the definitions previously applied to these perceptions do not accurately or adequately reflect the patients' experience and suggest that 'body perception disturbance' is an appropriate term to describe this phenomenon. Findings suggest a complex interaction between pain, body perception disturbance and central remapping. Clinically, our findings support the use of treatments that target cortical areas, which may reduce body perception disturbance and pain. Further study is required to measure the extent of body perception disturbance and clarify the relationship with pain in CRPS. 
710 This study was supported by grants from the Depart711 ment of Health, Arthritis Research Campaign and The 712 Royal National Hospital for Rheumatic Diseases do713 nated funds. Particular thanks goes to the participants 714 for their immense contribution to this study and Dr. 715 Caroline Ellis-Hill for her contribution to thought 716 development.

\section{References}

718 Beck AT, Ward CH, Mendelson M, Mock J, Erbaugh J. An inventory for measuring depression. Arch Gen Psychiatry 1961;4:561-71.
Cleeland CS, Nakamura Y, Mendoza TR, Edwards KR, Douglas J, Serlina RC. Dimensions of the impact of cancer pain in a four country sample: new information from multidimensional scaling. Pain 1996;67:267-73.

Cleeland CS, Ryan KM. Pain assessment: global use of the Brief Pain Inventory. Ann Acad Med 1994;23:129-38.

Cutting J. Study of anosognosia. J Neurol Neurosurg Psychiatry 1978;41:548-55.

Denzin N, Lincoln Y. Collecting and interpreting qualitative materials 1998;vol. 3. California: Sage; 1998.

Devor M, Raber P. Heritability of symptoms in an experimental model of neuropathic pain. Pain 1990;42:51-67.

Devor M. Comment from the Editor. Pain, In press, Corrected proof.

Flick U. An introduction to qualitative research. London: Sage; 2002.

Flor H. Cortical reorganisation and chronic pain: implications for rehabilitation. J Rehabil Med 2003;35:66-72.

Forderreuther S, Sailer U, Straube A. Impaired self-perception of the hand in complex regional pain syndrome (CRPS). Pain 2004;110:756-61.

Galer BS, Butler S, Jensen MP. Case reports and hypothesis: a neglectlike syndrome may be responsible for the motor disturbance in reflex sympathetic dystrophy (Complex Regional Pain Syndrome1). J Pain Symptom Manage 1995;10:385-91.

Galer BS, Jensen M. Neglect-like symptoms in complex regional pain syndrome: results of a self-administered survey. J Pain Symptom Manage 1999;18:213-7.

Glaser BG. The constant comparative method of qualitative analysis. In: McCall GJ, Simmons JL, editors. Issues in participant observation. Reading, Mass: Addison Wesley; 1969. p. 216-28.

Glaser BG. Theoretical sensitivity. Mill Valley, California: The Sociology Press; 1978.

Graziano MSA, Botvinick MM. How the brain represents the body: insights from neurophysiology and psychology. Common Mech Percept Action 2002;19:136-57.

Lewis J, McCabe C, Shenker N, Blake D. Experiences of complex regional pain syndrome: a case of neglect? Rheumatology 2003;42(1):22.
Love AW, Grabsch B, Clarke DM, Bloch S, Kissane DW. Screening for depression in women with metastatic breast cancer: a comparison of the Beck Depression Inventory Short Form and the Hospital Anxiety and Depression Scale. Aust N Z J Psychiatry 2004:38.

Maihofner C, Handwerker HO, Neundorfer B, Birklein F. Patterns of cortical reorganization in complex regional pain syndrome. Neurology 2003;61:1707-15.

Maihofner CA, Handwerker H, Neundorfer B, Birklein F. Cortical reorganization during recovery from complex regional pain syndrome. Neurology 2004;63:693-701.

McCabe CS, Haigh RC, Ring EF, Halligan PW, Wall PD, Blake DR. A controlled pilot study of the utility of mirror visual feedback in the treatment of complex regional pain syndrome (type 1). Rheumatology (Oxford) 2003;42:97-101.

Moseley GL. Graded motor imagery is effective for long-standing complex regional pain syndrome: a randomised controlled trial. Pain 2004;108:192-8.

Moseley GL. Graded motor imagery for pathologic pain. A randomized control trial. Neurology 2006;67:2129-34.

Moseley L. Distorted body image in complex regional pain syndrome. Neurology 2005;65:773.

Phillips KA. Body dysmorphic disorder: the distress of imagined ugliness. Am J Psychiatry 1991;148:1138-49.

Pleger B, Tegenthoff M, Ragert P, Forster A, Dinse H, Schwenkreis P, et al. Sensorimotor returning in complex regional pain syndrome parallels pain reduction. Ann neurol 2005;57:425-9.

Richards L. Using NVivo in qualitative research. Melbourne: QSR International; 2002.

Seale C. The quality of qualitative research. London: Sage; 1999.

Skrzypek S, Wehmeier PM, Remschmidt H. Body image assessment using body size estimation in recent studies on anorexia nervosa. A brief review. Eur Child Adolesc Psychiatry 2001;10:215-21.

\section{Computer Program}

SPSS. SPSS for windows (12.0.1). 2003. SPSS.

Stanley M, Cheek J. Grounded theory: exploiting the potential for occupational therapy. Br J Occup Ther 2003;66:143-50.

Stanton-Hicks M, Janig W, Hassenbusch S, Haddox JD, Boas R, 795 Wilson P. Reflex sympathetic dystrophy: changing concepts and 796 taxonomy. Pain 1995;63:127-33.

Strauss AL, Corbin J. Basics of qualitative research. Techniques and procedures for developing grounded theory. Thousand Oaks: Sage; 1998.

Wall PD, Devor M, Inbal JW, Scadding JW, Schonfeld D, Seltzer Z, et al. Autotomy following peripheral nerve lesions: experimental anaesthesia dolorosa. Pain 1979;7:103-13.

Ware JE, Sherbourne CD. The MOS 36-item short-form health survey (SF-36). Med Care 1992;30:473-83.

Wilkie IC, Dolan S, Lewis J, Blake DR. Autotomy: a terminological inexactitude. Pain In press, Corrected proof.

Zigmond AS, Snaith RP. The hospital anxiety and depression scale. 808 Acta Psychiatr Scand 1983;67:361-70. 